\title{
Full Arch Implant Restoration Using Fast and Fixed Protocol: A Case Report
}

\author{
Thapa $\mathrm{D}^{1}$, Shrestha $\mathrm{P}^{1}$ \\ ${ }^{1}$ Consultant Prosthodontist, Delight Dental Care, Kathmandu \\ ${ }^{2}$ Dental Surgeon, Delight Dental Care, Kathmandu
}

\begin{abstract}
Delivering a full arch implant restoration, which transits a patient from the frustrations of edentulism to the stability and function of a fixed prosthesis, is one of the most rewarding experiences in dentistry. With the advancement in digital technology, and with modified implant design and advance prosthetic options, what started as a two stage delayed loading procedure in the seventies, evolved to immediate implant placement in extraction sockets and even immediate loading of these implants in a growing number of indications.

In this case report, the same evolution "immediate implant placement and immediate loading techniques" has been described using fast and fixed protocol from Bredent to rehabilitate patient's failing dentition.

Key words: Full arch implant restoration; Immediate implant placement; Immediate Loading; Sky fast and fixed protocol
\end{abstract}

\section{Introduction}

$\mathrm{T}$ The concept of replacing an entire failing dentition with immediate implant placement and immediate/ early loading implant supported fixed prosthesis has been gaining wide clinical acceptance. Branemark's configuration proposed use of five implants on mandible and six on maxilla to support complete arch fixed prosthesis, with all implants placed anteriorly parallel to each other and splinted together by a passively fitted prosthesis. ${ }^{1}$ However, placing these multiple implants parallel is a great challenge, especially when patient has limited bone and does not prefer regenerative procedures.

Intentionally inclined implants have been proposed as an alternate to grafting. ${ }^{2,3,4,5,6}$ This

Conflict of Interest: No

\section{*Corresponding Author}

Dr. Dipak Thapa, MDS

Consultant Prosthodontist, Delight Dental Care, Kathmandu

E-mail:drdthapa@icloud.com helps to avoid sinus antrum in the maxilla and prevents conflict with mandibular nerve. ${ }^{3}$ These techniques not only assist in reducing the length of cantilevers and improve the antero-posterior distribution of implants around an arch but also reduce the number of implants. ${ }^{4,5}$ Thus, reducing the cost of the treatment and making it affordable to greater number of edentulous or nearly edentulous patients.

Initial implant stability is a prerequisite for immediate function. ${ }^{3}$ Though, the micro textured implant surface create favorable conditions for enhanced osseointegration, it is macroscopic implant design that influences the most for implant initial stability. ${ }^{7}$ Self taping thread, increased diameter and length, modification of number of threads, type of threads and taper of the body of implants are the modifications that increase bone implant contact and should be considered during implant selection for immediate loading. ${ }^{8}$

Multi unit abutments are designed to rehabilitate both edentulous and partially edentulous arches, particularly when using it in clinically 
and scientifically proven "all on four" or similar treatment concept with tilted implants. Regardless of the system, multi unit abutment and its accessories standardizes and simplifies the restorative process thus allows the dentist to pursue the very best prosthesis that modern technology has to offer.

\section{Case Report}

A 65 years female patient was referred to the dental clinic for prosthodontic treatment. She had multiple teeth missing in both maxillary and mandibular arches $(18,17,22$ to 27,33 to 42 , and 47); all the maxillary remaining teeth (16 to 21) including lower right canine (43) and first premolar (44) were severely periodontal compromised. Her lower left premolars (34 \& 35) and molars $(36,37,38 \& 48)$ were satisfactory. Her lower right first molar (46) and second premolar (45) fractured few days back, which were the only teeth with which she could chew. Now these were painful and she immediately needed prosthodontic intervention. (Fig 1-3)

After a complete history and patient examination, impressions were made for diagnostic casts and patient was advised for OPG (Fig 4). Few tentative treatment plans were made and discussed with the patient. She opted for implants supported fixed prosthesis with immediate or early temporization. Then patient was advised for CBCT of maxilla and mandible and details of both surgical and prosthetic plan was worked out. Five days prophylactic antibiotic was started one day before the surgery.

On the first day of surgery, tooth $46,45,44$ and 43 were atraumatically extracted under local anesthesia. Sockets were thoroughly cleaned and curetted. Four implants (Bredent, Sky Implant System, Germany) were placed, three on the extraction sockets $(46,44 \& 43)$ and one on the healed site (33) (Fig 5). The insertion torque of all the implants were more than $30 \mathrm{Ncm}$, which is required for immediate loading. Bone graft material (Geistlich, Bio Oss) was used to fill gaps in the sockets and around implants on position 46 and 44 . Four straight multi unit abutments were selected and torqued to $25 \mathrm{Ncm}$ on each implant. Healing abutments were hand tightened on them and interrupted sutures were placed. An abutment level impression using closed tray transfer was made for temporary fabrication.

On the second day of surgery (after two days), all the remaining maxillary teeth were extracted, flap reflected and alveoloplasty was done to reduce the height of anterior maxilla (Fig $6 \&$ 7). The patient had high lip line with gummy smile and protruded premaxilla, so to shift the transition line above smile line, about 2-3 mm of bone were trimmed using large round surgical bur. Six implants (Bredent, blueSky system) were placed after the osteotomy. Two distal most implants on each side were placed tilted, just anterior and parallel to the anterior wall of sinus, to avoid sinus lift. Except the left distal most implant, all the implants achieved torque higher than $30 \mathrm{Ncm}$. Bone graft material (Bio Oss, Geistlich) was used for socket preservation and to fill the jumping gap between the sockets and anterior implants. Multiunit abutments were selected and torqued to $25 \mathrm{Ncm}$. Healing abutments were placed and suturing was done. Abutment level impression was made for temporary prosthesis fabrication.

After two days, the patient was called for the first set of temporary restoration. The healing abutments were removed and the prepared temporary restorations were attached. X-rays of implant superstructure systems were performed. The fit of lower temporary was not satisfactory so prosthetic copings were separated form the restoration and picked up in the patient mouth using Qu-resin (Bredent, pink color). Occlusion was adjusted and both temporary restorations were torqued to $10 \mathrm{Ncm}$ (Fig 8). The maxillary 
implant with low primary stability was not included in the temporary restoration. Sutures were removed after 10 days.

After a month, with satisfactory soft tissue healing, second set of temporaries were fabricated and screwed for better esthetic. This time for maxillary arch, porcelain fused to metal ceramic bridge was made (Fig 9).

Before proceeding for final prosthesis, remaining mandibular teeth were prepared. Full arch maxillary and mandibular impressions were made on abutment platform level, open tray technique using prosthetic coping rather the impression coping, using one step putty wash technique (Zhermack Elite HD+) (fig 10). Master models were prepared using abutment analogue and Gingifast Elastic (Zhermack) (fig 11). Temporary prosthesis were installed to the master model and used to verify the cast as well as to mount the cast to the articulator (Bio Art A 7 plus) (Fig 12).

Porcelain fused to metal bridges were fabricated as final prosthesis (Fig $13 \& 14$ ). Occlusion, aesthetics and phonetics were verified during porcelain trail (bisque stage).
To confirm the passive fit, the prosthesis was bonded to the prosthetic copings in the patient's mouth rather than on the cast (cement-screw retained prosthesis). ParaCore dual cured resin system (Coltene) was used for this purpose. After bonding procedure, the prosthesis was unscrewed, all excess cement trimmed and polished and after cleaning, screwed back to $15 \mathrm{Ncm}$ to the abutment. The screw access holes were filled with temporary materials. The crowns on natural teeth were luted with resin modified glass ionomer cement (GC-Fujicem).

Minor occlusion adjustments were done using articulating paper. Simultaneous bilateral contacts on all posterior teeth, posterior disocclusion on protrusion, and unilateral group function were confirmed. This was rechecked and refined on subsequent appointments as well. Finally, access holes were closed using Teflon as base and flowable composite resin (Fig 15-17).

The patient was followed on 1,2,3 and 6 months post loading and implant stability and soft tissue health were assessed which was completely healthy at follow up time (Fig 18).
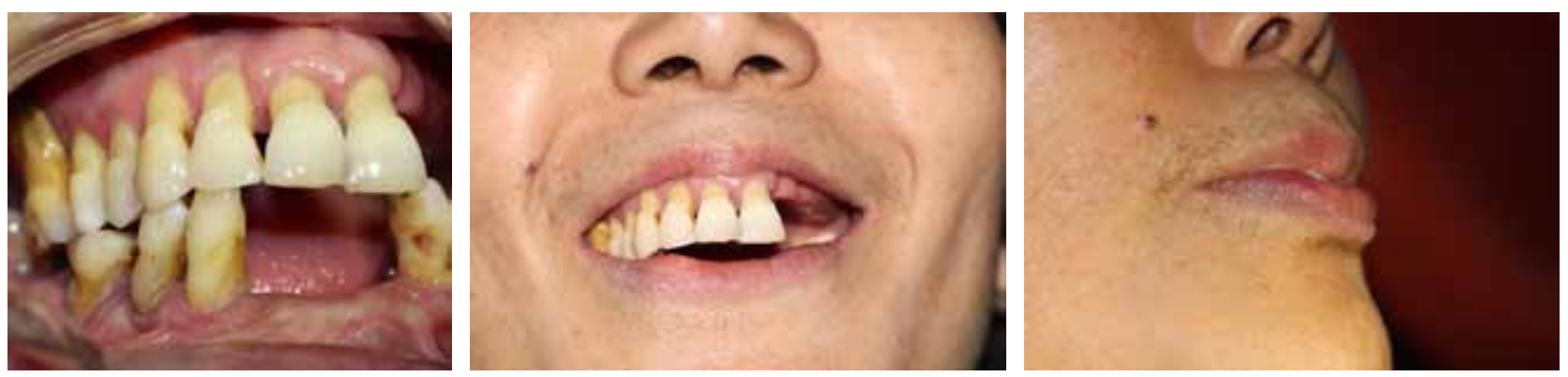

Figure 1,2,3: Pre Op

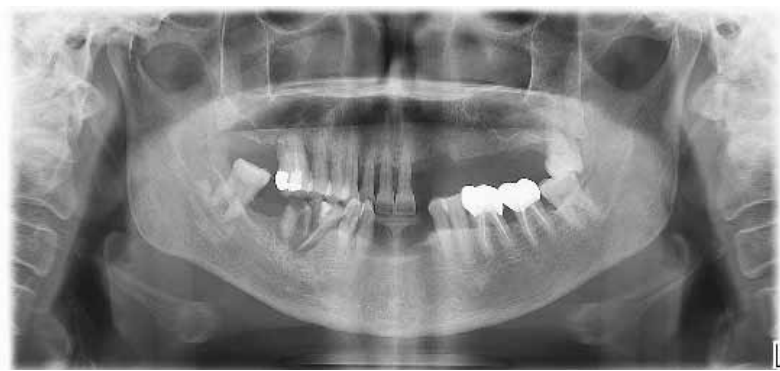

Figure 4: $O P G$ 


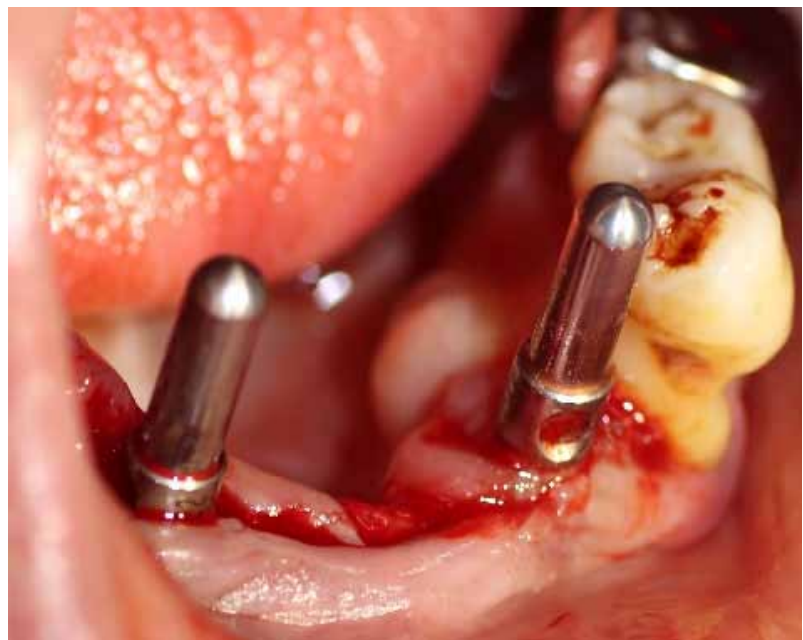

Figure 5: Implant pilot drilling, mandibular arch

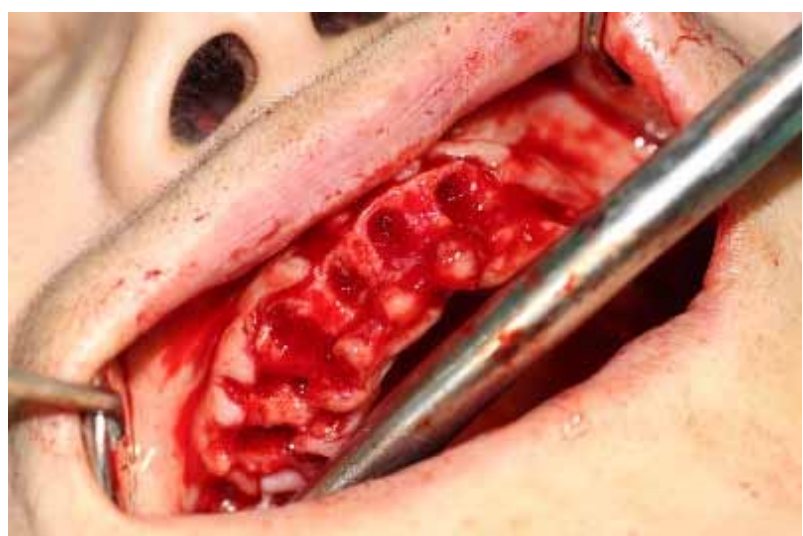

Figure 7: Alveoplasty, anterior maxillary arch

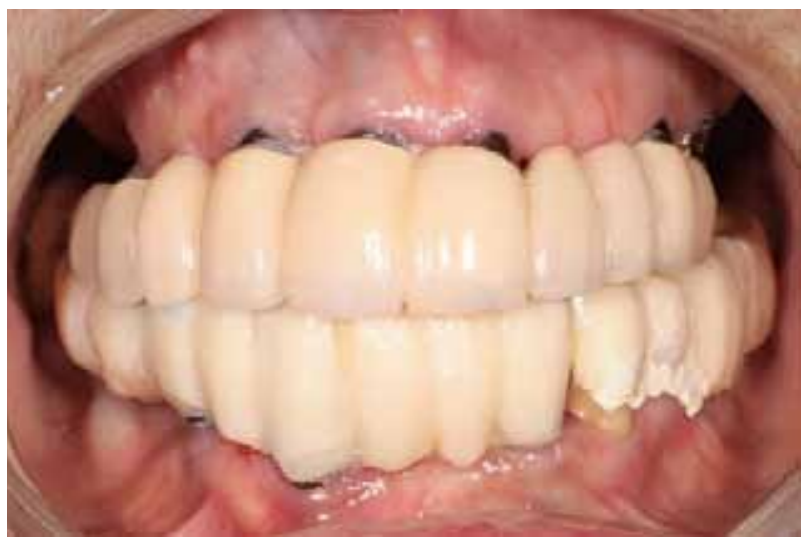

Figure 9: Second temporary

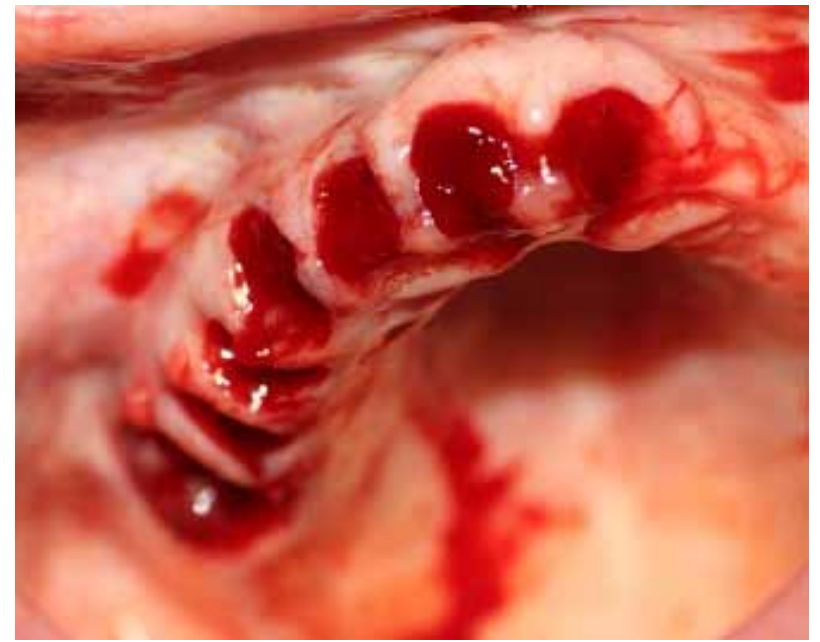

Figure 6: After extraction, maxillary arch

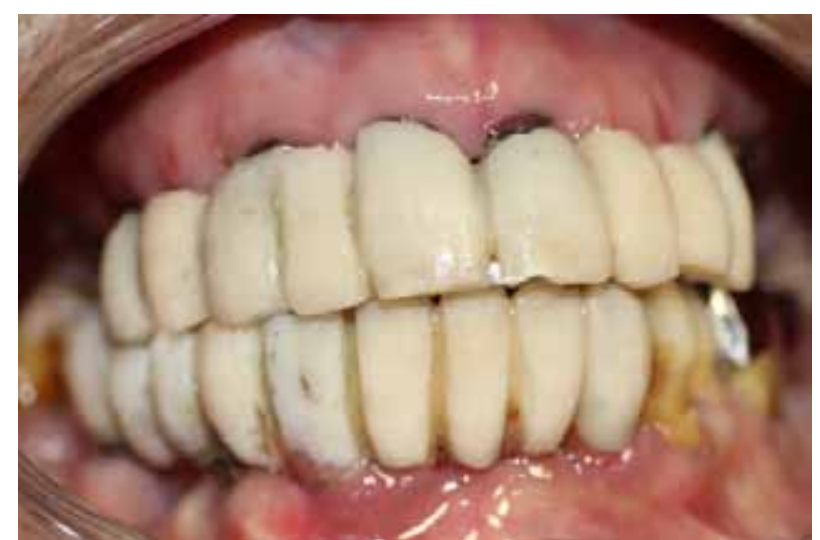

Figure 8: Provisional restorations

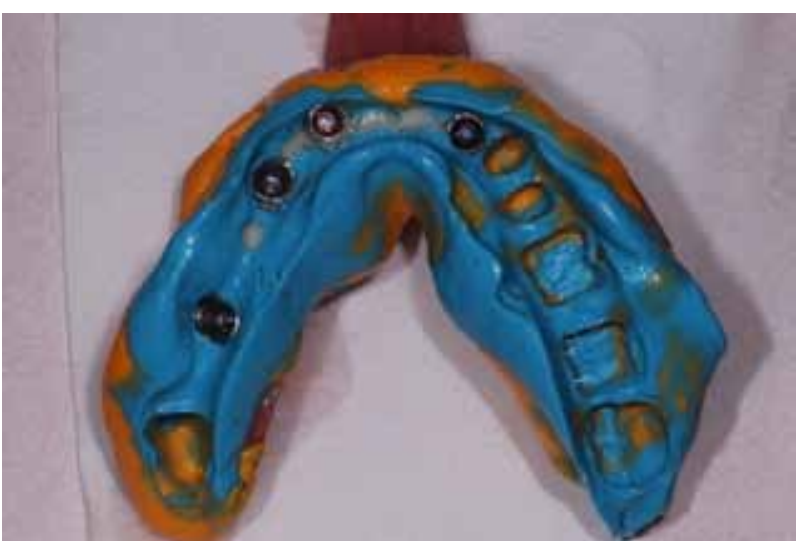

Figure 10: Mandibular impression 


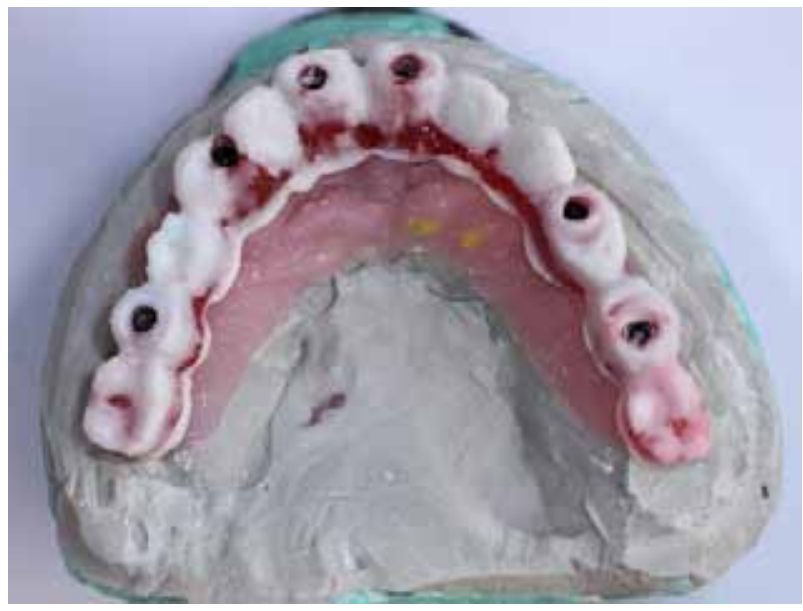

Figure 11: Maxillary master cast with wax pattern

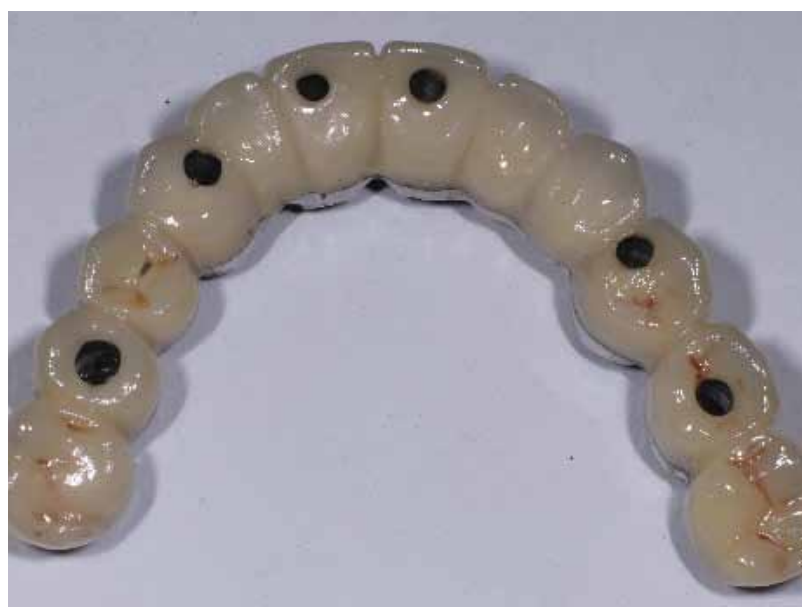

Figure 13: Maxillary final restoration

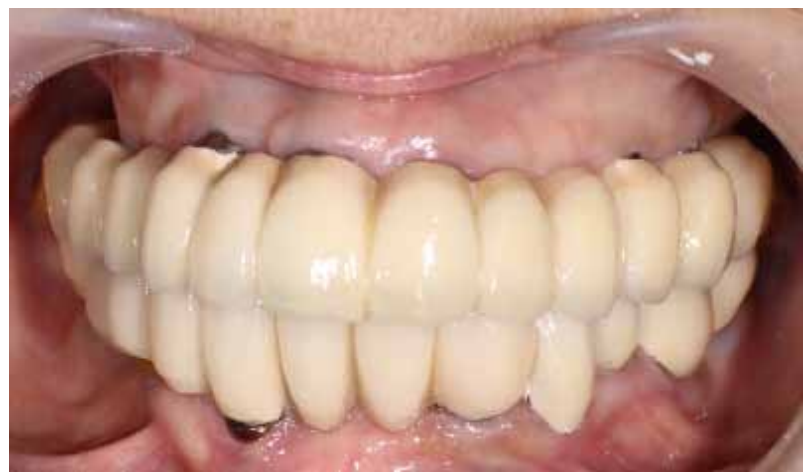

Figure 15: Final restorations in MIP

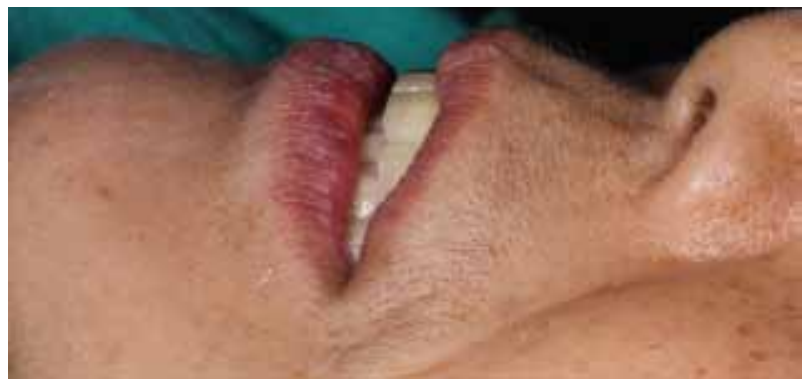

Figure 17: Lip support

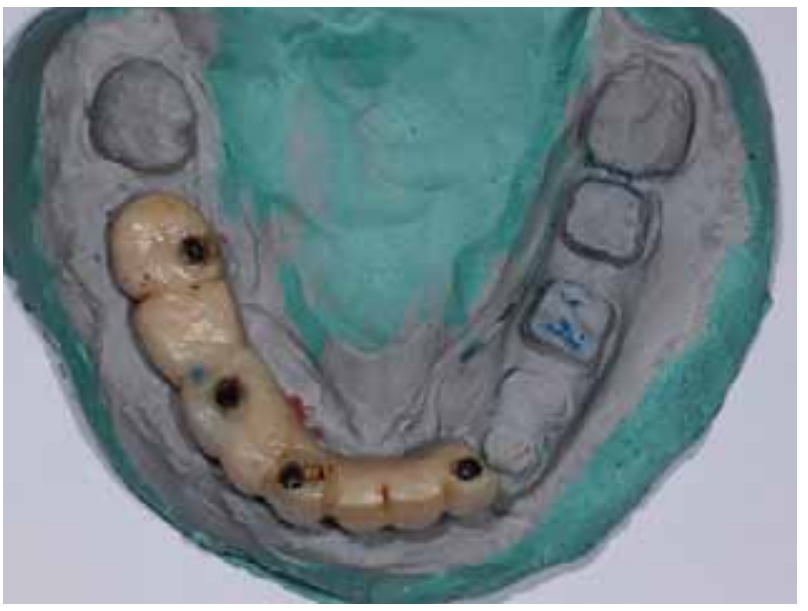

Figure 12: Mandibular provisional restorations

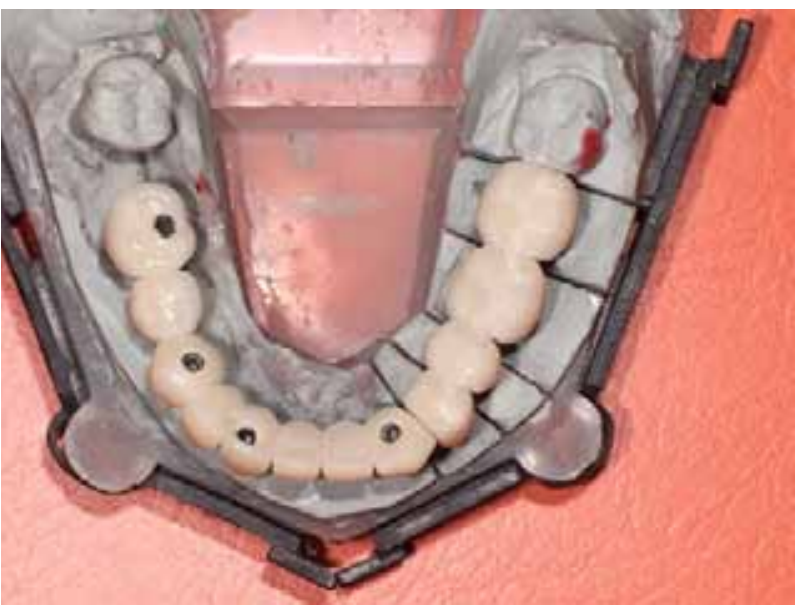

Figure 14: Mandibular final restorations

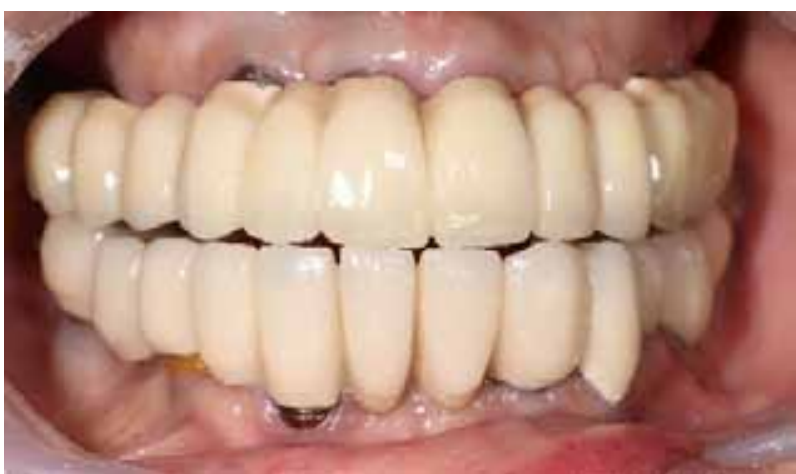

Figure 16: Final restorations in protrusion

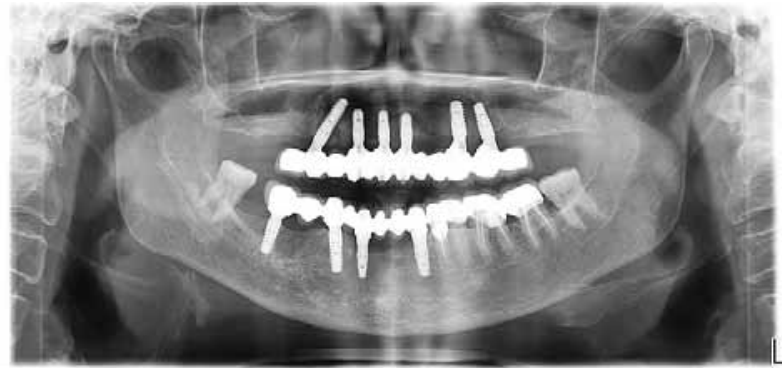

Figure 18: OPG 


\section{Discussion}

The final outcome of the treatment relies on careful diagnosis and meticulous planning. Cone beam-computed tomography (CBCT) has made significant contribution to the planning and has become mandatory in such cases. But with the advancement in digital technology, virtual implant planning (both the prosthetic and surgical phase) and executing that plan using of $3 \mathrm{D}$ printed surgical guide is becoming a trend now days.

Regarding the number of implants for full arch prosthesis, there are various recommendations; mostly 4-6 implants on mandible and 6-10 on mandible. ${ }^{1,10}$ Fast and fixed protocol recommends minimum of 6 Sky implants on maxilla and 4 on mandible.

Bredent Sky implants are conical cylindrical with double thread till apex and have self-taping compression threads, ideal design for achieving high primary stability thus immediate or early loading possible with shortened healing time. ${ }^{11}$

For the angulation of the posterior implant, an angulation aid showing 35 degree is available. Tilting implants further than this increases strain magnitude around implants thus not recommended for biomechanical reasons. ${ }^{6,12}$ Two tilted implants placed in a curved arch once cross arch splinted, using angled abutments, decreases the stresses around the distal implants. ${ }^{12,13}$

Multi unit abutments are used to correct varying heights among the implants, thus a level restorative platform among multiple implants can be established. Angled multi unit abutments (17.5 and 35 degrees) are used to correct the divergence of implants and create a unified path of insertion for the final prosthesis. They are also used as one time abutment concept. All additional prosthetic steps are carried out using copings at abutment level.
Regarding final prosthesis there are various options with combination of materials and technologies such as metal frame, pink acrylic and denture teeth(hybrid denture); milled high performance polymer (HPP) frame and composite teeth bonded with resin; milled monolithic or layered Zirconia; and the latest one with milled titanium or $\mathrm{CoCr}$ alloy frame veneered with milled individual restorations (Toronto bridge). They all can be veneered with pink composite or pink ceramic for pink aesthetic. ${ }^{14}$

In this case we opted for traditional long span metal ceramic restoration (FP1\& FP2 type prosthesis) for easy designing and ease for maintaining hygiene.

\section{Conclusions}

Delivering a full arch implant restoration to edentulous or nearly edentulous patient is definitely a wonderful experience both for the clinician and the patient however it requires tremendous amount of hard work and dedication. Understanding the details of surgical and prosthetic protocol, in depth planning, good lab support and the most of all patient's motivation is a pre-requisite to success.

\section{References}

1. Branemark, Per-Ingvar(1977). Osseointegrated Implants in the Treatment of Edentulous Jaw. Sweden: Almquivist \&Weksell International. pp 79, 81,83.

2. Fortin Y, Sullivan RM, Rangert BR. The Marius implant bridge: surgical and prosthetic rehabilitation for the completely edentulous upper jaw with moderate to severe resorption: a 5-year retrospective clinical study. Clin Implant Dent Relat Res. 2002;4(2):69-77. doi: 10.1111/ j.1708-8208.2002.tb00155.x. PMID: 12121606.

3. Maló P, Nobre Mde A, Petersson U, Wigren S. A pilot study of complete edentulous rehabilitation with immediate function using a new implant design: case series. Clin Implant Dent Relat Res. 2006;8(4):223-32. doi: 10.1111/j.17088208.2006.00024.x. PMID: 17100748. 
4. Krekmanov L, Kahn M, Rangert B, Lindström $\mathrm{H}$. Tilting of posterior mandibular and maxillary implants for improved prosthesis support. Int J Oral Maxillofac Implants. 2000 MayJun;15(3):405-14. PMID: 10874806.

5. Aparicio C, Perales P, Rangert B. Tilted implants as an alternative to maxillary sinus grafting: a clinical, radiologic, and periotest study. Clin Implant Dent Relat Res. 2001;3(1):39-49. doi: 10.1111/j.1708-8208.2001.tb00127.x. PMID: 11441542 .

6. Mattsson T, Köndell PA, Gynther GW, Fredholm U, Bolin A. Implant treatment without bone grafting in severely resorbed edentulous maxillae. J Oral Maxillofac Surg. 1999 Mar;57(3):281-7. doi: 10.1016/s02782391(99)90673-0. PMID: 10077198.

7. Buser D, Schenk RK, Steinemann S, Fiorellini JP, Fox $\mathrm{CH}$, Stich $\mathrm{H}$. Influence of surface characteristics on bone integration of titanium implants. A histomorphometric study in miniature pigs. J Biomed Mater Res. 1991 Jul;25(7):889-902. doi: 10.1002/ jbm.820250708. PMID: 1918105.

8. Calandriello $\mathrm{R}$, Tomatis $\mathrm{M}$, Rangert $\mathrm{B}$. Immediate functional loading of Brånemark System implants with enhanced initial stability: a prospective 1- to 2-year clinical and radiographic study. Clin Implant Dent Relat Res. 2003;5 Suppl 1:10-20. doi: 10.1111/j.17088208.2003.tb00011.x. PMID: 12691646.

9. Daudt Polido W, Aghaloo T, Emmett TW, Taylor TD, Morton D. Number of implants placed for complete-arch fixed prostheses: A systematic review and meta-analysis. Clin Oral Implants Res. 2018 Oct;29 Suppl 16:154-183. doi: 10.1111/clr.13312. PMID: 30328199.

10. Isidor F. Influence of forces on peri-implant bone. Clin Oral Implants Res. 2006 Oct; 17 Suppl 2:8-18. doi: 10.1111/j.1600-0501.2006.01360.x. PMID: 16968378.

11. Begg T, Geerts GA, Gryzagoridis J. Stress patterns around distal angled implants in the all-on-four concept configuration. Int J Oral Maxillofac Implants. 2009 JulAug;24(4):663-71. PMID: 19885406.

12. Taruna M, Chittaranjan B, Sudheer N, Tella S, Abusaad M. Prosthodontic perspective to allon-4® concept for dental implants. J Clin Diagn Res. 2014 Oct;8(10):ZE16-9. doi: 10.7860/ JCDR/2014/9648.5020. Epub 2014 Oct 20. PMID: 25478475; PMCID: PMC4253293.

13. Wong N. All-on-X: Final Fixed Prostheric Options for Full Arch Implant Cases. Oral Health, Nov 92017. 